\title{
Segmentation Method for Pathological Brain Tumor and Accurate Detection using MRI
}

\author{
Khurram Ejaz ${ }^{1}$, Mohd Shafry Mohd Rahim ${ }^{2}$, Amjad Rehman $^{3}$, Huma Chaudhry ${ }^{4}$, Tanzila Saba ${ }^{5}$, \\ Anmol Ejaz ${ }^{6}$, Chaudhry Farhan $\mathrm{Ej}^{7}$ \\ ${ }^{1,2,4}$ Faculty of Computing, UTM, Johor Bahru, Malaysia \\ ${ }^{3}$ Faculty of CCIS, Al Yamamah University, Riyadh, Saudi Arabia \\ ${ }^{5}$ Department of Info. Sys., Prince Sultan University, Riyadh, Saudi Arabia \\ ${ }^{6}$ Faculty of Allied Sci, UOL, Lahore, Pakistan \\ ${ }^{7}$ Dept. of Engineering, Nokia, R\&D, Alkhobar, Saudi Arabia
}

\begin{abstract}
Image segmentation is challenging task in field of medical image processing. Magnetic resonance imaging is helpful to doctor for detection of human brain tumor within three sources of images (axil, corneal, sagittal). MR images are nosier and detection of brain tumor location as feature is more complicated. Level set methods have been applied but due to human interaction they are affected so appropriate contour has been generated in discontinuous regions and pathological human brain tumor portion highlighted after applying binarization, removing unessential objects; therefore contour has been generated. Then to classify tumor for segmentation hybrid Fuzzy K Mean-Self Organization Mapping (FKM-SOM) for variation of intensities is used. For improved segmented accuracy, classification has been performed, mainly features are extracted using Discrete Wavelet Transformation (DWT) then reduced using Principal Component Analysis (PCA). Thirteen features from every image of dataset have been classified for accuracy using Support Vector Machine (SVM) kernel classification (RBF, linear, polygon) so results have been achieved using evaluation parameters like Fscore, Precision, accuracy, specificity and recall.
\end{abstract}

Keywords-Brain tumor; level set; Hybrid Fuzzy K Mean (Hybrid FKM); Discrete Wavelet Transformation (DWT); Scalable Vector Machine (SVM); Magnetic Resonance Image (MRI); Principal Component Analysis (PCA)

\section{INTRODUCTION}

MRI gives internal visualization of soft tissues of brain and analysis if MRI is from plentiful visual information when expert when wants to examine brain for detection of brain tumor. Two kind of brain tumor have been seen in images like benign and second one is malignant. Experts check type of tumor with boundary of tissue in MRI. Three orientation of MRI are available for visualization like Sagittal (x axis), coronal ( $\mathrm{Y}$ axis) and Axil ( $\mathrm{Z}$ axis). In this paper Axil slice of T2 give more highlight of tumor boundary but challenge to detect due to homogenous intensity. Experts interested observing brain tumor from digital images which are noisier. To identify information from these digital images, the process of segmentation has been used. Manual segmentation is much time consuming if the volume of image becomes high. Therefore, automatic segmentation using different method becomes important and challenging for more accurate detection. Segmentation improves with combination of thirteen texture and statistical features, reduction of features and classification to segment target labels.

In Section II detailed related work along critical table is discussed, in Section III detail analysis of two datasets is shown. Section IV is for methodology with stepwise implementation, Section $\mathrm{V}$ is results and discussion, and at last Section VI provides conclusion and future directions.

\section{RELATED WORK}

MRI imaging is compromising due to noise, complexity of detection of brain tumor disease [21]. Patient declares normal or abnormal with analysis of MR imaging. The importance of MR imaging has been seen with plentiful visualization of soft tissues [22]. MR imaging needs improvement due to signal to noise ratio [23] and they need enchantment so segmentation method diagnoses brain tumor in MR imaging and they classifies tumor as malignant or benign.

For detection of brain tumor, MR imaging incorporated by manual segmentation, semi-automatic segmentation and automatic segmentation. Manual segmentation is performed with some software's, but they have issue of variation every time. Semi-automatic segmentation has affected from personal intervention whereas automatic segmentation is incorporating with special knowledge for detection of brain tumor [24]. Special kind of filters has been used for detection of brain tumor [25]. Automatic detection is only made for local region [26]. With using combination of WFRFT+PCA+SVM achieve maximum accuracy [27].

Many studies have been found for detection of brain using classification for purpose of segmentation and results can be seen with some accurate results.

Various novel system for detection of brain tumor has used Magnetic Resonance imaging (MRI) modality [6], [15]. Using none supervised learning Fuzzy $\mathrm{C}$ mean, $\mathrm{K}$ mean for automatic segmentation are more focused for specificity and sensitivity [18], [19]. Classification techniques are more focused for accurate detection of brain tumor [1]-[6]. From methodology point of view classification is important for feature extraction [20]. Determined symmetric or none symmetric features are modelled using preprocessing and post processing techniques for pathological brain detection [6][10]. Feature Extraction of Brain MRI is transforming [1], In 
DWT, it converts into digital values whereas in SWT can see brain features more accurately. Magnetic Resonance Brain Image has been classified based on Adaptive Chaotic PSO [2]. Combined three different techniques to find best classification for detection of normal brain or abnormal brain so applied DWT+PCA+ ADCPSO (Adaptive comparative particle swarm optimization) and after that neural network has been applied for best results. The classification results were 98.5 per cent with combination of ADCPSO+FNN over 160 images from Harvard site. The advantage of this technique was better result achievement and disadvantage of this paper is neural network working is unclear. Magnetic Resonance Brain Image Classification by AN Improved Artificial BEE Colony Algorithm for classification(ACB)[3], various classification techniques for MRI to detect brain either normal or abnormal has applied and it has been proved ACB better as compare to GA (Genetic Algorithm), Differential Evolution (DE) and PSO. For classification, it has been performed ACB (Artificial BEE colony) worse for training of FNN so it has been decided performance of optimization depend upon application, so devised Scalable Chaotic Artificial Bee Colony algorithm classifies normal and abnormal brains of $\mathrm{T} 1$ weight images with accuracy of 100 percent. Devised novel hybrid classifier for detection of normal and abnormal brain and has obtained 100 percent accuracy after performing experiments [4]. Before experiment has applied series of step over image like wavelet transformation at different levels then PCA for feature extraction then finally applied BNN and accurate result has found. Achieved accuracy after using combination of DWT, PCA, KSVM and kernel which has been used is GRB kernel and it accuracy is 99.38 over Axial images [5]. SVM + PSO over trained data and then optima; optimal KSVM told about normal or abnormal brain and found 97.7 per cent accuracy which is larger amount as compare to BP-NN(86.22\%), RBF$\mathrm{NN}(91.33 \%)$ [6]. Modern classification of brain MRI images and that's that classifications are "WFRFT+PCA+GEPSVM", "WFRFT+PCA_TSVM, the proposed results are better than other eight classes[7]. Feed forward neural (FNN) had been used as classifier for optimization and in novel work has been combined with biograph based optimization and particle swarm optimization in way of training FNN, finally achieved proposed classification up to 100 percent with combination of WE(Wavelet Entropy and HBP(hybridization BO,PSO) [8]. Contribution was proved that "DWPT+TE+GEPSVM+RBF method gave better classification than other state of art methods or techniques" and accuracy was 100 per cent [9]. Successful for invention of automatic abnormal brain detection using improved classifier with combination of QPSO, KSVM and wavelet energy. In paper conducted experiment obtained best results comparatively. Secondly wave let energy feature is best for abnormal brain detection. [10]. The disadvantage of this work is, this is very complex work. The good thing is that his accuracy was 97.78 percent with technique of BBO-KSVM [13]. Robustness has been defined as human visuals image with abnormality and system confirms the abnormality like system did in 0.002 second to prove robustness. Technique over 25 normal images were selected whereas rest of 25 were abnormal .90 per cent correct identification [11].

Segmentation methods are important for segmenting of MRI image and they may be fast if threshold values are assigned. Model based techniques which are based on geometric deform model they use full automatic segmentation but computationally expensive but it calculates sensitivity of tumor boundaries [12]. Performed automatic segmentation after converting knowledge into probabilistic values in image so find complex feature and Neural Network convolution is applied because it can learnt more complex features in MRI therefore multi modal proposed for MRI [13]. Segmentation using morphological operation like feature of brain tumor centroid $\mathrm{X}$, centroid $\mathrm{Y}$, area and in comparison segmentation technique using morphological is better than other rest of methods [14]. 2D adoption noise removed, segmentation is achieve with removing strong speckle and enhance the weak boundaries of medical image, the weakness is accomplished with range filter to segment various anatomical structure [15]. In first generation segmentation methods are threshold methods, region growing methods and edge-based detection and in second generation they are cluster, classifier, deformation model and graph cluster. In third generation, graph guided approach, shape model, appearance model, medical image segmentation methods, algorithm and application. [16]. Segmentation methods are important for detection of brain tumor when MRI is compromising different issues during their process. Combined $\mathrm{K}$ mean cluster algorithm with fuzzy $\mathrm{C}$ scan in minimal execution time in four stages like preprocessing, clustering, feature extraction and validation from noisy portion of MRI [17]. An un supervised method with a clustering approach for tumor identification and tissues segmentation in magnetic resonance is important from un supervised perspective [18]. Two techniques hybrid Fuzzy c mean, $\mathrm{k}$ mean using SOM achieved best sensitivity, mean square root, specificity over Harvard repository images and has achieved best segmentation over three sources of image like axil images, corneal and sagittal [28]. Vector classification and $\mathrm{Z}$ indexing [19] is good paper for classification point of view of classification and feature can be Efficient Feature detection of image using Multimedia database using Query [20]. Contextual, fuzzy classification methods, robust features and extraction methods achieve good results during segmentation [32]-[36].

The segmentation of brain tumor has been performed in continuous regions as well as in discontinuous regions. Brain tumor is as a feature and has been detected in MRI when applies quad tree for detection of region of interest (ROI) in continuous region [21]. Quad tree active contour level set are compromising in discontinuous regions. Hybrid FKM-SOM technique has filled gap of partitioning of tumor portion and edema region, but limitation occurs due to intensities mixing, analyzed three sources of images like Axil, Sagittal and Corneal but segmentation results using comparison parameter are not sufficiently proving for segmentation and evolution of brain tumor. 
TABLE I. ACCURACIES OF SEGMENTATION

\begin{tabular}{|c|c|c|}
\hline Paper title & Techniques & $\begin{array}{l}\text { Segmentation } \\
\text { Accuracy }\end{array}$ \\
\hline $\begin{array}{l}\text { (Sneha Dhurkunde } \\
\text { 2016) }\end{array}$ & $\begin{array}{l}\text { Histogram, Fuzzy c mean, K } \\
\text { mean }\end{array}$ & 79.5 \\
\hline $\begin{array}{l}\text { (Saleha Masood*, } \\
\text { 2015) }\end{array}$ & $\begin{array}{l}\text { Thresholding, Region growing, } \\
\text { Clustering, Classifiers, Bayesian } \\
\text { approach, Deformable methods, } \\
\text { atlas guided approach, edge } \\
\text { based methods, compression } \\
\text { based method }\end{array}$ & Missing \\
\hline $\begin{array}{l}\text { (Norouzi et al., } \\
\text { 2014) }\end{array}$ & $\begin{array}{l}\text { Thresholding, histogram, Region } \\
\text { of interest, Clustering techniques, } \\
\text { Classification techniques, } \\
\text { Expectation maximization, } \\
\text { Graph Cut }\end{array}$ & Missing \\
\hline (Rajaei et al., 2012) & Image texturing, Range filters & Missing \\
\hline $\begin{array}{l}\text { (Padma and } \\
\text { Sukanesh, 2011) }\end{array}$ & Dominant grey level run length & 85 \\
\hline $\begin{array}{l}\text { Vishnuvarthanan et } \\
\text { al., 2016). }\end{array}$ & $\begin{array}{l}\text { Hybrid Fuzzy k mean-SOM, } \\
\text { Fuzzy c mean, neural network, } \\
\text { evaluation matrix }\end{array}$ & 91 \\
\hline $\begin{array}{l}\text { (Abdel-Maksoud et } \\
\text { al., 2015) }\end{array}$ & $\begin{array}{l}\text { Median filter, K mean cluster, } \\
\text { Fuzzy c mean }\end{array}$ & 87.5 \\
\hline $\begin{array}{l}\text { Pei, L., Reza, S. M., } \\
\text { and Iftekharuddin, } \\
\text { K. M. (2015) }\end{array}$ & $\begin{array}{l}\text { K cluster, histogram, joint label } \\
\text { fusion }\end{array}$ & 71 \\
\hline $\begin{array}{l}\text { Yang, G., Zhang, } \\
\text { Y., Yang, J., Ji, G., } \\
\text { Dong, Z., Wang, S., } \\
\text { et al. (2016) }\end{array}$ & $\begin{array}{l}\text { Classification, Pattern } \\
\text { Recognition, Support Vector } \\
\text { Machine, Biogeograph based } \\
\text { optimisation }\end{array}$ & Missing \\
\hline $\begin{array}{l}\text { Zhang, Y., Dong, } \\
\text { Z., Wang, S., Ji, G., } \\
\text { and Yang, J. (2015) }\end{array}$ & $\begin{array}{l}\text { Shannon entropy; Tsallis } \\
\text { entropy; discrete wavelet packet } \\
\text { transform, support vector } \\
\text { machine kernel technique, } \\
\text { pattern recognition; classification }\end{array}$ & Missing \\
\hline $\begin{array}{l}\text { (Sudharani et al., } \\
\text { 2016) }\end{array}$ & $\begin{array}{l}\text { Sampling, histogram, } \\
\text { morphological operation }\end{array}$ & 89 \\
\hline
\end{tabular}

Issues are arisen in brain tumor using MRI using segmentation when brain spread in discontinuous regions, due human less interactivity especially when MRI is nosier so to separate tumor tissues from normal tissues with accuracy become challenge. Accuracy of with combination of different segmentation method can be seen in Table I.

\section{MATERIAL}

\section{A. Data Set}

Fig. 1 gives view of Scope of work from dataset no. 1, Two datasets have been based analyzed like Harvard data set and one local hospital MRI data set. Data set 1 is based on three orientation for single patient of MRI. Dataset number 1 is consisting clinical used MR images dataset and it is consisting of eleven total patients; patient class has been divided into two main classes and their names are malignant or Benign. According to history of patient we have seven malignant patients and four Benign patients. Both classes are for of abnormal patients. One patient is further divided into three kind of images plane like corneal (y axis plan), sagittal ( $\mathrm{x}$ axis plane) and $\mathrm{z}$ axis plane which is known as axil plane. Dataset is consisting of $\mathrm{T} 2$ sequences. $\mathrm{T} 2$ sequence images are more enhance so that radiologist can easily observe before operation and treatment

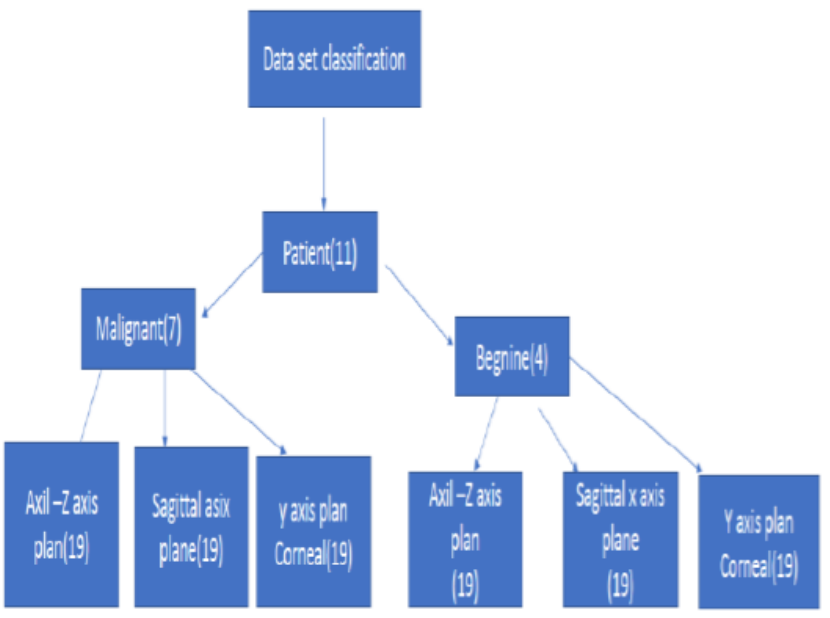

Fig. 1. Proposed feature diagram for data set number

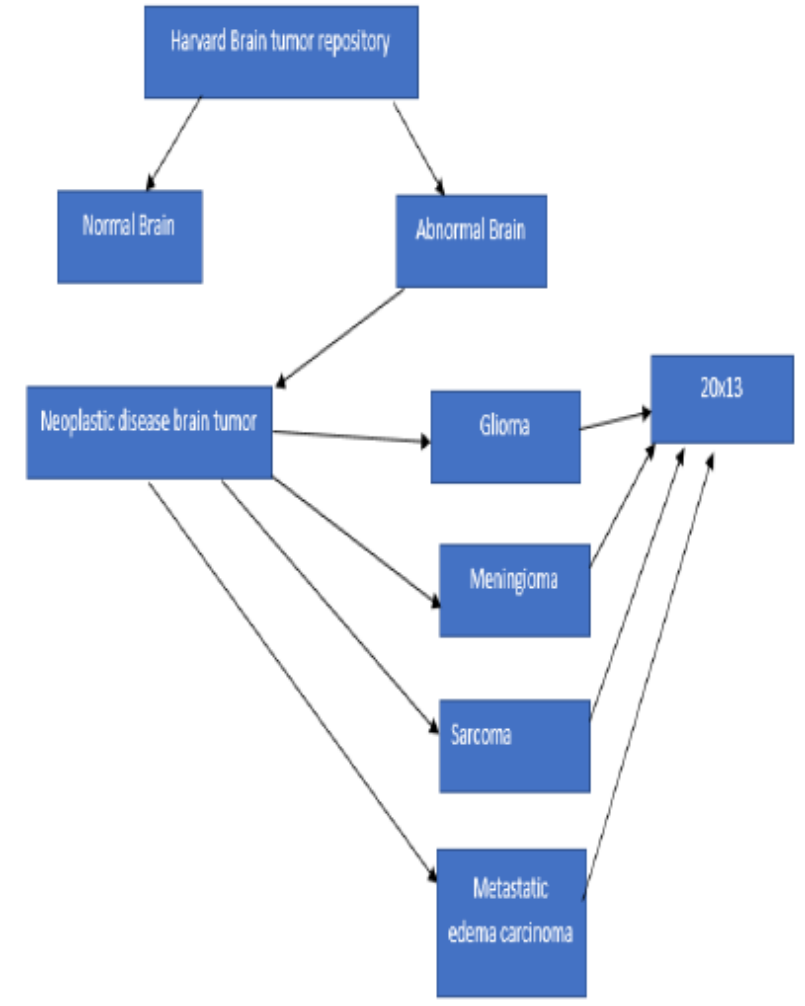

Fig. 2. Data set number 2 diagram 

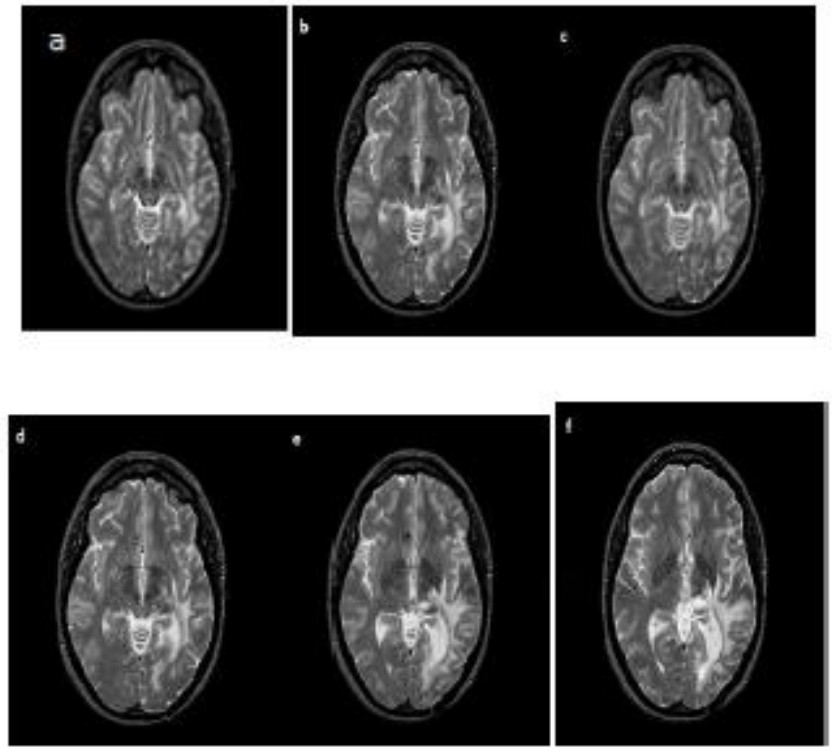

Fig. 3. Harvard dataset used by Y Zhang dataset (2).

Fig. 2 is from benchmark dataset from Harvard brain published repository. The bench mark paper used Harvard repository of axial images [5]. Same wise [5], same orientation of dataset used same by [7] and are performing experiment over axil Z-axis images for accurate identification of brain tumor. These images are MR images and novel accurate has been evaluated for identification of brain tumor so it's one view of images and for these images output accuracy of classification is more than 90 per cent. Other planes are compromising. In data set result of classification is not mentioned.

Datasets images has been taken for same patient, all of axis plane data set like in Fig (a), (b), (c), (d), (e), and (f) for same patient.
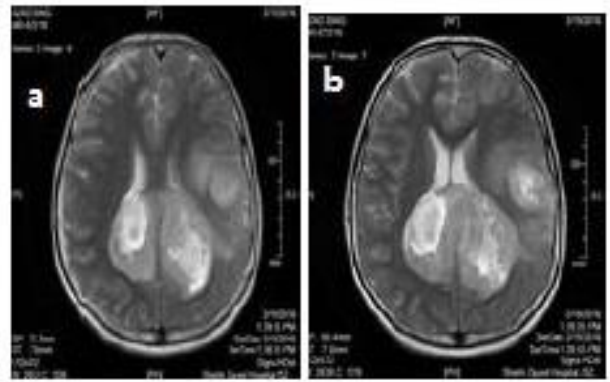

(Dataset 1 Axil image for $\mathrm{x}$ axis plane)

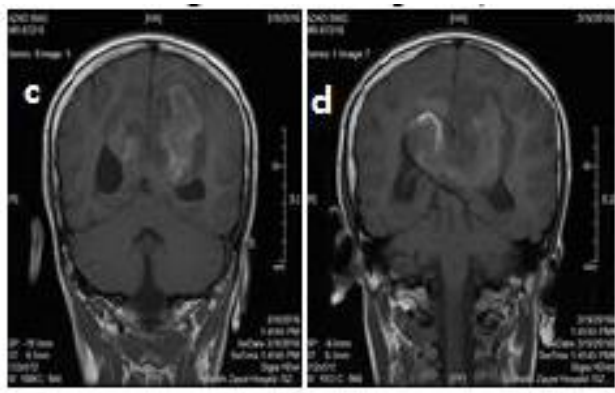

(Dataset 1 Corneal images y axis plane)

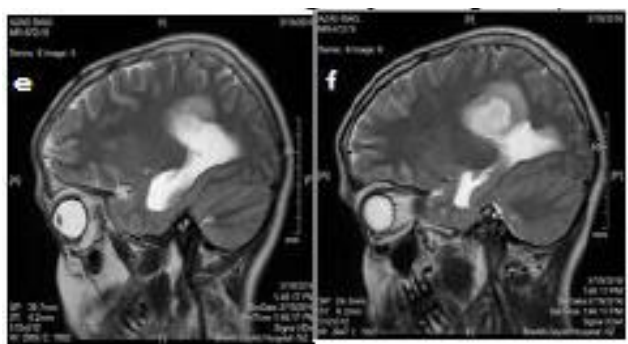

(Dataset 1 sagittal $\mathrm{x}$ axis plane)

Fig. 4. Fig. a, Fig. b, Fig. c, Fig. d, Fig. e, Fig. f for x, y, z-axis plane.

Above Fig. 3 and 4 are from two datasets like clinical dataset and another Harvard published dataset.

\section{METHODOLOGY}

The methodology in Fig. 5 shows ten steps, and Fig. 6, 7, 8 for contour and Fig. 9 and 10 for DWT and Fig. 11 for SVM classification. In first step dataset has been input, in step two dataset has been preprocessed for noised reduction, contour has been initiated in step three for detection of tumor location, in step number 4 segmented portion achieved, in step number 5 features of image using DWT have been extracted for accurate detection, in step number sixth achieved features have been reduced using PCA, in step number seventh 13 features have been achieved, in step number eight flavor of KSVM(Linear, polygon) achieved accuracy of classification and at second last step evaluation of accuracy achieved through specificity, accuracy, Fscore, precision and Recall have been derived and last one are results so comparison of dataset can be achieved.

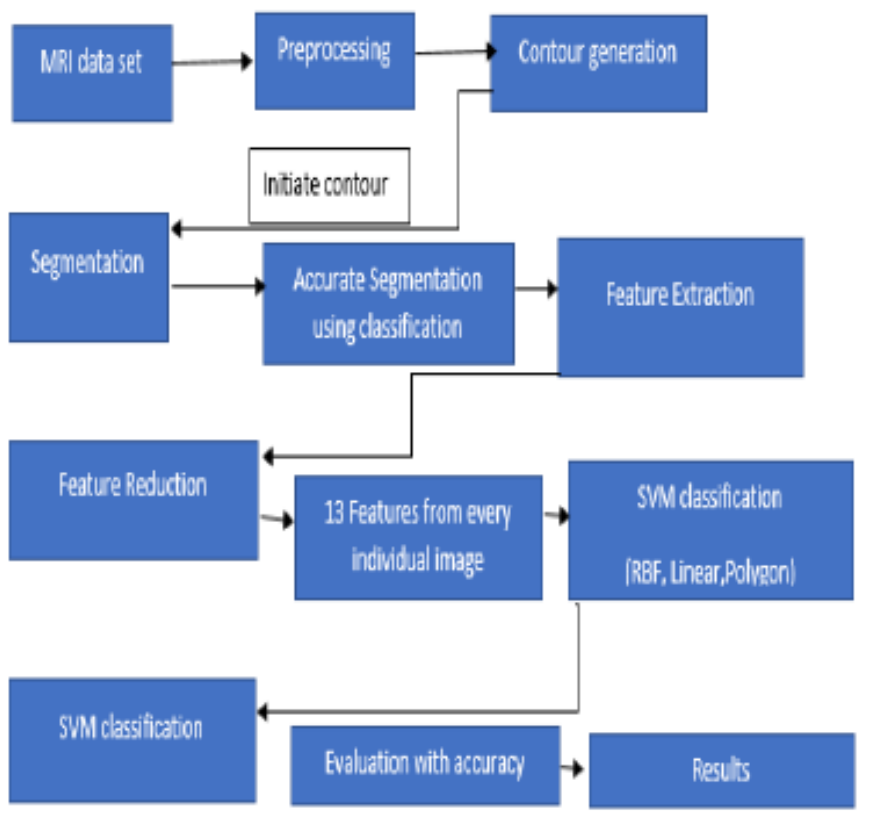

Fig. 5. Frame work

Image segmentation performed with level set function the contour level zero so has been chosen manually [32]. Segmentation done without selection of proper initiate contour (IC) effects the segmentation results for discontinuous tumor within MRI. Therefore, in proposed strategy proper contour 
has been generated, firstly binarization [31] has been performed seconding remove un essential then contour map have been generated for detection of discontinuous tumor portion as feature discontinuously region of interest. From Fig. 6 and 7 contour map has been generated.

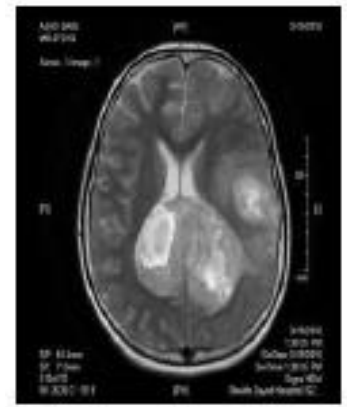

Fig. 6. Original image DS1.

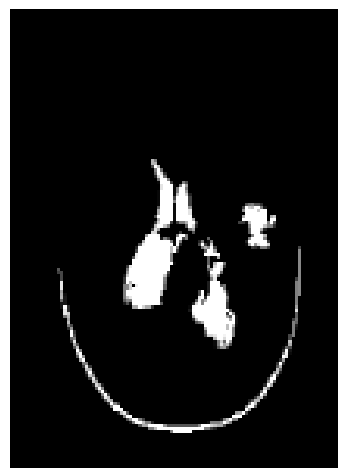

Fig. 7. Binarization Fig. (6).

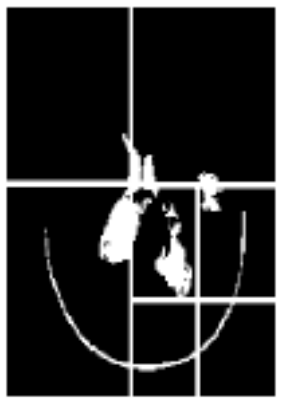

Fig. 8. Initiated Contour map generated

DWT (Discrete Wavelet transformation) is applying, image is considering as a signal and presents in time domain. [29] In time domain image has been divided locally into bands so energy level of image features has been highlighted.

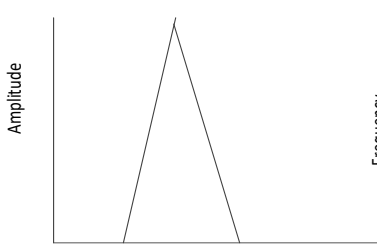

Frequency
Time

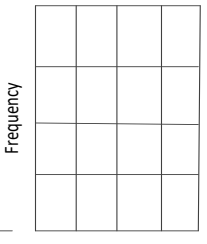

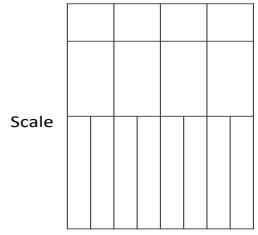

Time
Fig. 9. DWT signal representation

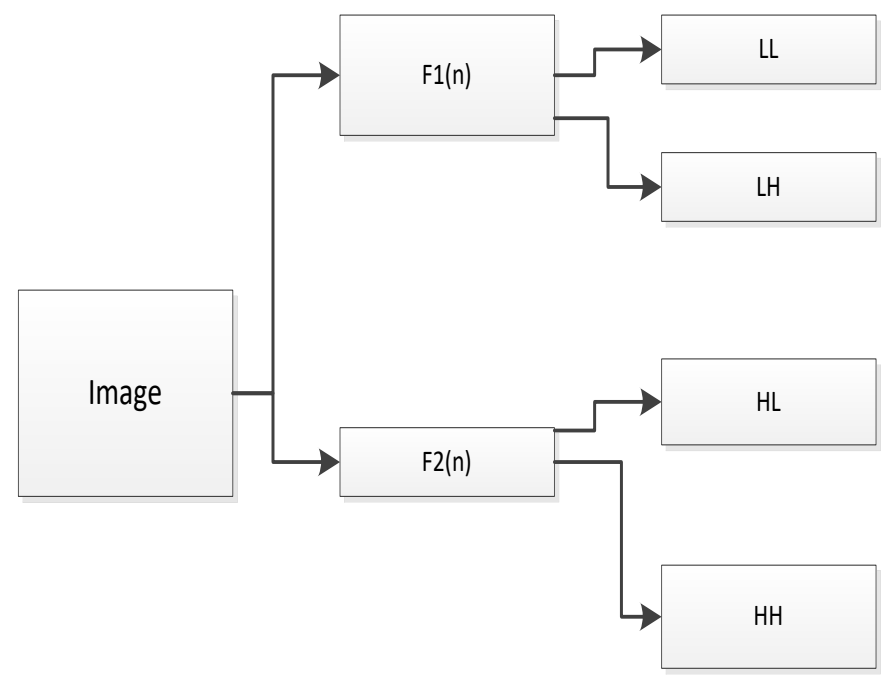

Fig. 10. DWT signal representation with high pass and low pass variations.

In step number 6, PCA (Principal Component Analysis) performs for dimension reduction of image features. Firstly, identifies uncorrelated elements from image then least un correlated element remove from data set images so the group of most corelated elements have been obtained. Fig. 9 to 10 shows DWT representation of image.

Step number 8 is accurate feature classification, obtained elements are classified through Support Vector Machine(SVM) like two classes are [-1,1]. SVM gives hyper plane and in such plan classified output(relevant) classes of images in query [30].

$$
\mathrm{F}(\mathrm{x}, \mathrm{y})=\left\{\left(\mathrm{x}_{\mathrm{n}}, \mathrm{y}_{\mathrm{n})} / \mathrm{x}_{\mathrm{n}} \in \mathrm{R}^{\mathrm{p}}, \mathrm{y}_{\mathrm{n}} \mathcal{E}(1,-1)\right\}\right.
$$

To address above two issues firstly I have tested my dataset which is only Axil ( $\mathrm{z}$ axis images) image so I could see one picture of dataset then I have trained my dataset using DWT, PCA, SVM and Kernel (Fig. 11) based SVM and next I have proposed dataset with aim of accurate classification of all of three kind images.

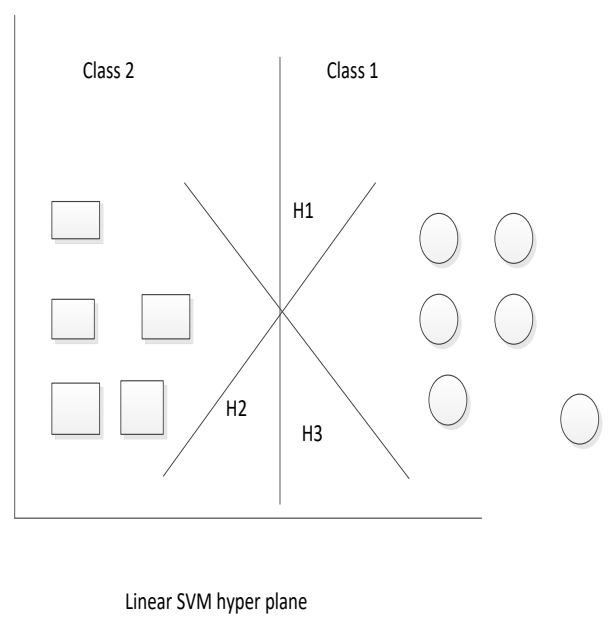

Fig. 11. KSVM has variation of SVM like RBF kernel, quadratic kernel and linear kernel for accurate detection of features in queried image. 
TABLE II. REFERENCED BASED 13 FEATURES TABLE

\begin{tabular}{|c|c|}
\hline Feature & Reference \\
\hline contrast & $\begin{array}{l}\text { Zhang, Y., and Wu, L. } \\
\underline{(2012)}\end{array}$ \\
\hline corelation & $\begin{array}{l}\text { Zhang, Y., Ji, G., Yang, J., } \\
\text { Wang, S., Dong, Z., } \\
\text { Phillips, P., et al. (2016) }\end{array}$ \\
\hline energy & $\begin{array}{l}\text { Zhang, Y., and Wu, L. } \\
\underline{(2012)}\end{array}$ \\
\hline homogenity & $\begin{array}{l}\text { Zhang, Y., Wang, S., Dong, } \\
\text { Z.,Phillip, P., Ji, G., and } \\
\underline{\text { Yang, J. }(2015 b)}\end{array}$ \\
\hline mean & $\begin{array}{l}\text { (Abdel-Maksoud } \text { et al., } \\
\text { 2015), (Vishnuvarthanan et } \\
\text { al., 2016) }\end{array}$ \\
\hline standard deviation & $\begin{array}{l}\underline{\text { S., Ji, G., and Yang, J. }} \\
\underline{(2015)}\end{array}$ \\
\hline root mean square & $\begin{array}{l}\text { (Abdel-Maksoud et al., } \\
\text { 2015), (Vishnuvarthanan et } \\
\text { al., 2016) }\end{array}$ \\
\hline variance & $\begin{array}{l}\underline{\text { S., Ji, G., and Yang, J. }} \\
\underline{(2015)}\end{array}$ \\
\hline kurtosis & $\begin{array}{l}\underline{\text { S., Ji, G., and Yang, J. }} \\
\underline{(2015)}\end{array}$ \\
\hline skewness & $\begin{array}{l}\text { Zhang, } Y \text {., and } W u, L \text {. } \\
\underline{(2012)}\end{array}$ \\
\hline inverse difference movement & $\begin{array}{l}\text { Zhang, Y., Ji, G., Yang, J., } \\
\text { Wang, S., Dong, Z., } \\
\text { Phillips, P., et al. (2016) }\end{array}$ \\
\hline
\end{tabular}

Above Table II is list of features which we have used for accurate segmentation.

The pseudo code of our work is in nine steps and state flow In Fig. 12, Step wise construction can be seen for segmentation of tumor with methods and accuracy has been checked with flavor of kernels.

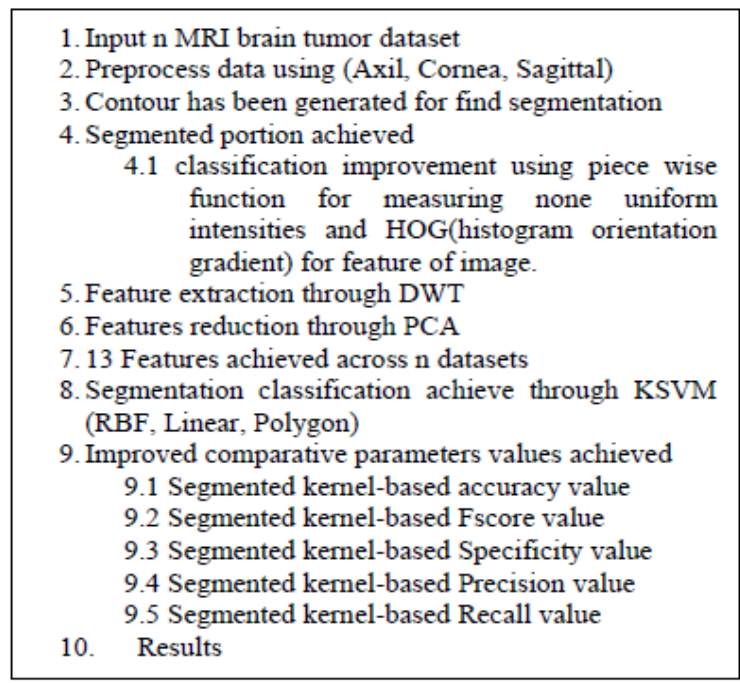

Fig. 12. Segmentation with step wise construction.

\section{RESULTS AND DISCUSSION}

In this paper, two data sets have been checked. Dataset number 1 is taken from sheikhzaidh hospital and second data set MR images have been taken from Harvard medical school repository.

Data set number 1, which is consisting of MR images, MR images are three sources like Axil, Corneal and Sagittal. For axil in Fig. 13 and Table III, corneal in Fig. 14 and Table IV and sagittal with comparison to bench mark in Table $\mathrm{V}$ and Fig. 15. In dataset number 1, eleven patients have been checked and for each patient 99x13(axil), 99x13(sagittal) and 99x13(corneal) has been overlooked and detected segmented tumor with accuracy and RBF, linear and polygon kernels whereas dataset number 2 which is consisting of only axil images for 42 years old patient. Same 13 features have been selected so total axil images are 20x13. The target labels are 0 or 1 . Zero mean malignant however 1 means benign. Same linear, polygon kernel, RBF and linear have been used for accurate detection. Fig. 12 and Table II are from bench mark dataset.

In this section using SVM three kernels, their names are polygon kernel, RBF and linear and their results can be seen kernel versus comparison evaluation parameters like accuracy, specificity, precision, recall and FScore. Experiment is performed over clinical dataset and over public data. In Fig. 13 a graph figure, benchmark dataset accuracy of segmentation can be seen across evaluation metrices. Fig. 14 is giving evaluation of Axil orientation segmentation accuracy whereas Fig. 15 gives picture of accuracy of coronal images. Fig. 16 the last one is showing picture of accuracy of sagittal along comparative analysis among these three orientations.

TABLE III. HARVARD BENCH MARK DATASET DATA SET NUMBER2 USING RBF, LINEAR AND LINER KERNEL

\begin{tabular}{|l|l|l|l|l|l|}
\hline & $\begin{array}{l}\text { Zhong et al } \\
\text { Accuracy }\end{array}$ & Specificity & Precision & Recall & Fscore \\
\hline $\begin{array}{l}\text { Linear } \\
\text { kernel }\end{array}$ & 66.6666667 & 66.66666667 & 66.66667 & 66.66667 & 66.66667 \\
\hline RBF kernel & 50 & 33.33333333 & 66.66667 & 50 & 57.14286 \\
\hline $\begin{array}{l}\text { Polynomial } \\
\text { Kernel }\end{array}$ & 33.3333333 & 66.66666667 & 0 & 0 & 0 \\
\hline
\end{tabular}

Table III is targeting Harvard data set and three kernels (Linear, RBF, Polynomial) have been checked across for evaluation.

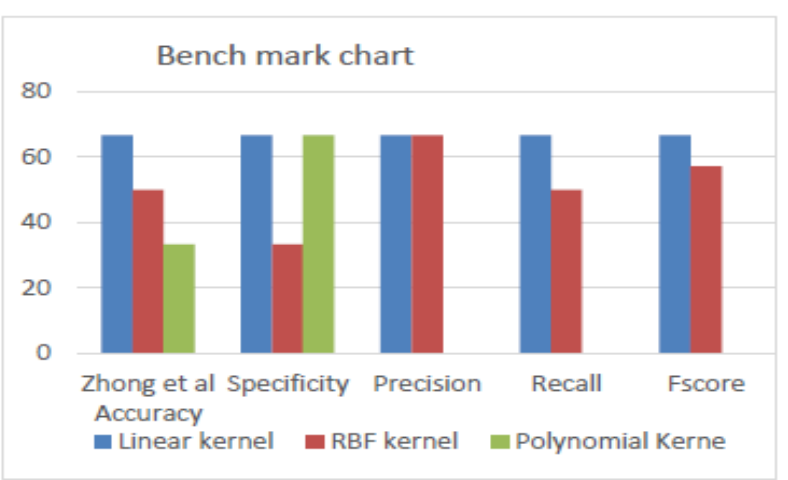

Fig. 13. Comparative chart 
TABLE IV. DATASET NUMBER 1AXIL IMAGES ACCURACY

\begin{tabular}{|l|l|l|l|l|l|}
\hline \multicolumn{6}{|l|}{ Accuracy of Axil images with Dataset1 } \\
\hline & $\begin{array}{l}\text { Accurac } \\
\text { y SZ }\end{array}$ & $\begin{array}{l}\text { Specificit } \\
\text { y }\end{array}$ & $\begin{array}{l}\text { Precisio } \\
\text { n }\end{array}$ & $\begin{array}{l}\text { Recal } \\
\text { I }\end{array}$ & $\begin{array}{l}\text { Fscor } \\
\text { e }\end{array}$ \\
\hline Linear & 66.666 & 95 & 10 & 50 & $\begin{array}{l}16.66 \\
7\end{array}$ \\
\hline RBF & 73.33 & 100 & 20 & 100 & 33.33 \\
\hline $\begin{array}{l}\text { Polynomi } \\
\text { al }\end{array}$ & 73.33 & 80 & 60 & 60 & 60 \\
\hline
\end{tabular}

Table IV is depict accuracy of clinical dataset number I across evaluation parameters.

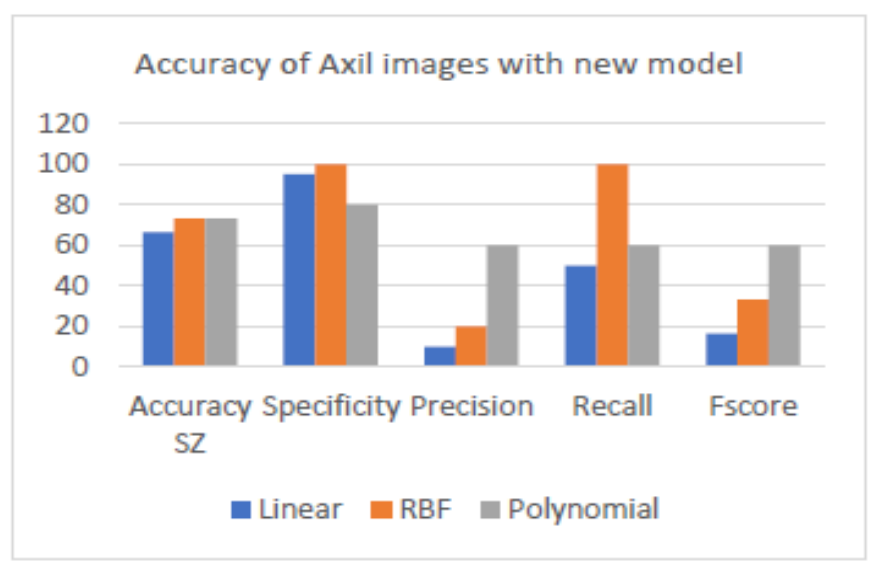

Fig. 14. Graph for axil images from dataset number 1

TABLE V. DATASET NUMBER 1 ACCURACY RESULTS WITH RBF, POLYGON AND LINEAR KERNEL

\begin{tabular}{|l|l|l|l|l|l|}
\hline \multicolumn{7}{|c|}{ Accuracy of Corneal images with Dataset no. 1 } \\
\hline & $\begin{array}{l}\text { Accuracy } \\
\text { SD } \\
\text { corneal }\end{array}$ & Specificity & Precision & Recall & Fscore \\
\hline Linear & 80 & 100 & 40 & 100 & 57.14286 \\
\hline RBF & 96.66667 & 95 & 100 & 90.90909 & 95.2381 \\
\hline Polynomial & 90 & 85 & 100 & 76.92308 & 86.95652 \\
\hline
\end{tabular}

Table $\mathrm{V}$ is giving accuracy of orientation of coronal using three kernels for accurate segmentation of tumor.

\section{corneal accuracy results with RBF, polygon and linear kernel}

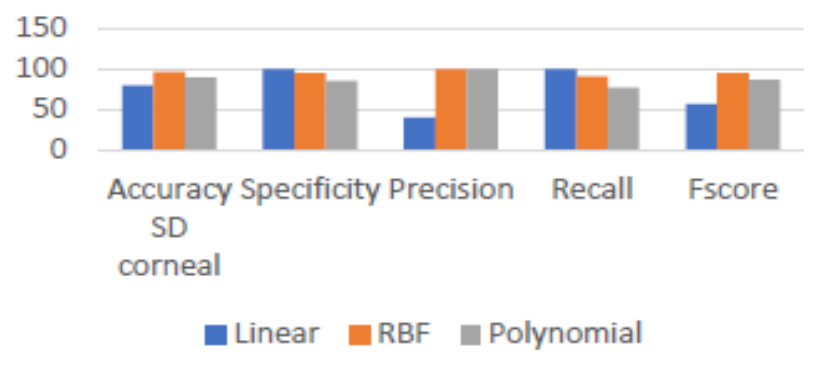

Fig. 15. Graph for Coronal images from dataset number 1
TABLE VI. TABLE FOR SAGITTAL IMAGES FROM DATASET NUMBER NO. 1 AND COMPARISON ACCURACY OF BOTH DATASETS

\begin{tabular}{|l|l|l|l|l|l|}
\hline \multicolumn{6}{|c|}{ Accuracy for comparative analysis of Dataset1 with bench mark dataset } \\
\hline & $\begin{array}{l}\text { Accuracy SZ } \\
\text { Sagittal }\end{array}$ & Specificity & Precision & Recall & Fscore \\
\hline Linear & 40 & 68.75 & 7.142857143 & 16.66666667 & 10 \\
\hline RBF & 53.33333333 & 100 & 0 & NaN & 0 \\
\hline Polynomial & 46.66666667 & 43.75 & 50 & 43.75 & 46.66667 \\
\hline
\end{tabular}

Table VI is for accuracy of kernels over dataset number 1 of sagittal ( $\mathrm{X}$ axis).

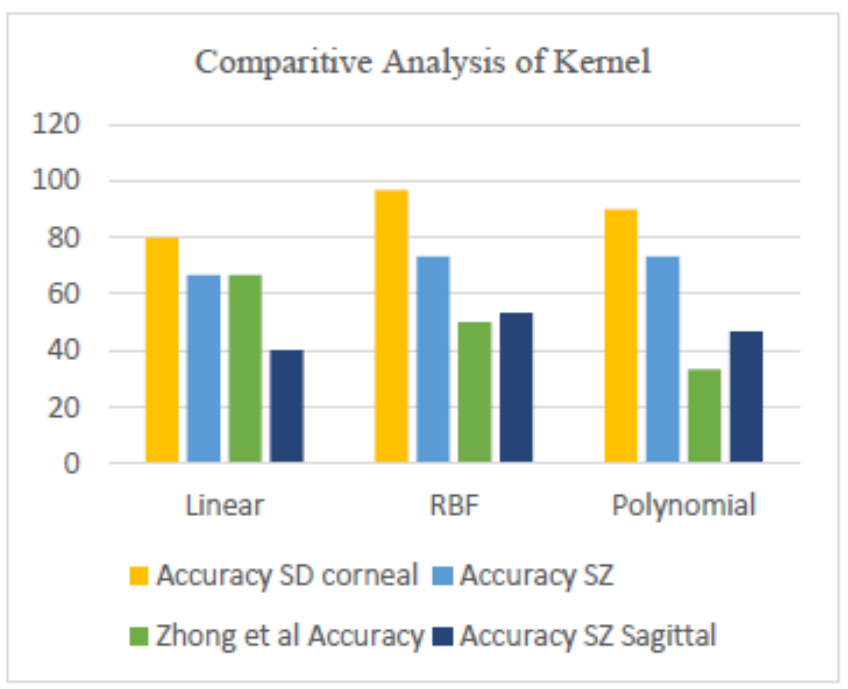

Fig. 16. For comparison of accuracy of both data set number 1 and dataset number 2

From above graph we can say clear cut difference of two dataset value for accurate segment detection of brain tumor using RBF, Linear and polynomial kernel. More transparent comparison has been drawn among datasets in above graph. Using linear kernel both datasets are equal whereas using RBF dataset 1 values need improvement and if using polynomial kernel then dataset 2 need more improvement as compare to dataset number 1 .

\section{CONCLUSION AND FUTURE WORK}

We have identified new improved scheme of steps of segmentation like appropriate contour generation, hybrid SOM-FKM for identification of tumor in discontinuous region and segmentation accurate results have been highlighted with evaluation parameters like accuracy, Fscore, precision and recall using RBF and polygon kernel where linear accuracy of dataset are almost 66.6 per cent whereas using RBF Harvard dataset used by Y Zhang accuracy is less than using dataset 1 so need to improve accuracy. In this paper, analyzed three sources of images (axil, corneal, sagittal) in first dataset whereas in second dataset (Harvard) has been only using axil images therefore future work will also to analyzed rest of source of images in dataset 2 . 


\section{REFERENCES}

[1] Zhang, Y., et al. Feature extraction of brain MRI by stationary wavelet transform. in Biomedical Engineering and Computer Science (ICBECS), 2010 International Conference on. 2010. IEEE.

[2] Zhang, Y., S. Wang, and L. Wu, A novel method for magnetic resonance brain image classification based on adaptive chaotic PSO. Progress In Electromagnetics Research, 2010. 109: p. 325-343.

[3] Zhang, Y., L. Wu, and S. Wang, Magnetic resonance brain image classification by an improved artificial bee colony algorithm. Progress In Electromagnetics Research, 2011. 116: p. 65-79.

[4] Zhang, Y., A hybrid method for MRI brain image classification. Expert Systems with Applications, 2011. 38(8): p. 10049-10053.

[5] Zhang, Y. and L. Wu, An MR brain images classifier via principal component analysis and kernel support vector machine. Progress In Electromagnetics Research, 2012. 130: p. 369-388Y.

[6] Zhang, Y., et al., An MR brain images classifier system via particle swarm optimization and kernel support vector machine. The Scientific World Journal, 2013. 2013.

[7] Zhang, Y.D., et al., Magnetic resonance brain image classification based on weighted-type fractional Fourier transform and nonparallel support vector machine. International Journal of Imaging Systems and Technology, 2015. 25(4): p. 317-327.

[8] Zhang, Y., et al., Pathological brain detection in magnetic resonance imaging scanning by wavelet entropy and hybridization of biogeography-based optimization and particle swarm optimization. Progress In Electromagnetics Research, 2015. 152: p. 41-58.

[9] Zhang, Y., et al., Preclinical diagnosis of magnetic resonance (MR) brain images via discrete wavelet packet transform with Tsallis entropy and generalized eigenvalue proximal support vector machine (GEPSVM). Entropy, 2015. 17(4): p. 1795-1813.

[10] Zhang, Y., et al., Preliminary research on abnormal brain detection by wavelet-energy and quantum-behaved PSO. Technology and Health Care, 2016. 24(s2): p. S641-S649.

[11] Gordillo, N., E. Montseny, and P. Sobrevilla, State of the art survey on MRI brain tumor segmentation. Magnetic resonance imaging, 2013. 31(8): .1426-1438.

[12] Yang, G., et al., Automated classification of brain images using waveletenergy and biogeography-based optimization. Multimedia Tools and Applications, 2016. 75(23): p. 15601-15617

[13] Dimililer, K. and A. İlhan, Effect of Image Enhancement on MRI Brain Images with Neural Networks. Procedia Computer Science, 2016. 102: p. 39-44.

[14] Işın, A., C. Direkoğlu, and M. Şah, Review of mri-based brain tumor image segmentation using deep learning methods. Procedia Computer Science, 2016. 102: p. 317-324.

[15] 1, S.D., Segmentation of Brain Tumor in Magnetic Resonance Images using Various Techniques. International Journal of Innovative Research in Science, Engineering and Technology, 2016.

[16] Rajaei, A., L. Rangarajan, and E. Dallalzadeh, Medical Image Texture Segmentation Usingrange Filter. computer science and information technology, 2012. 2(1).

[17] Masood, S., et al., A survey on medical image segmentation. Current Medical Imaging Reviews, 2015. 11(1): p. 3-14.

[18] Abdel-Maksoud, E., M. Elmogy, and R. Al-Awadi, Brain tumor segmentation based on a hybrid clustering technique. Egyptian Informatics Journal, 2015. 16(1): p. 71-81.

[19] Vishnuvarthanan, G., et al., An unsupervised learning method with a clustering approach for tumor identification and tissue segmentation in magnetic resonance brain images. Applied Soft Computing, 2016. 38: p. 190-212.

[20] Ejaz, K., A. Mateen, and I. Ehsan. Vector Shape Classification and Z indexing. in IEEE Intl. Conference on Intelligent Network and Computing ICINC. 2010.

[21] Kumar, E.P., Kumar, V.M. and Sumithra, M.G., 2013, July. Tumour detection in brain MRI using improved segmentation algorithm. In Computing, Communications and Networking Technologies (ICCCNT), 2013 Fourth International Conference on (pp. 1-7). IEEE.

[22] S. K. Nayak, Y. K., Dr. C. S. Panda. (2015). A Study on Brain Mri Image Segmentation Techniques International Journal of Research Studies in Computer Science and Engineering Volume 2, , PP 4-13

[23] Saleha Masood*, M. S., Afifa Masood, Mussarat Yasmin and Mudassar Raza (2015). A Survey on Medical Image Segmentation. Current Medical Imaging Reviews(Segmentation in medical imaging), 3-14

[24] Gordillo, N., Montseny, E., and Sobrevilla, P. (2013). State of the art survey on MRI brain tumor segmentation. Magnetic resonance imaging, 31(8), 1426-1438.

[25] Rajaei, A., Rangarajan, L., and Dallalzadeh, E. (2012). Medical Image Texture Segmentation Usingrange Filter. computer science and information technology, 2(1).

[26] Sudharani, K., Sarma, T., and Prasad, K. S. (2016). Advanced morphological technique for automatic brain tumor detection and evaluation of statistical parameters. Procedia Technology, 24, 13741387.

[27] Gonzalez, R. C., and Woods, R. E. (2005). Book on "Digital image processing": Prentice-Hall of India Pvt. Ltd.

[28] Vishnuvarthanan, G., Rajasekaran, M. P., Subbaraj, P., and Vishnuvarthanan, A. (2016). An unsupervised learning method with a clustering approach for tumor identification and tissue segmentation in magnetic resonance brain images. Applied Soft Computing, 38, 190212.

[29] Zhang, Y.D., et al., Magnetic resonance brain image classification based on weighted-type fractional Fourier transform and nonparallel support vector machine. International Journal of Imaging Systems and Technology, 2015. 25(4): p. 317-327.

[30] Yang, G., et al., Automated classification of brain images using waveletenergy and biogeography-based optimization. Multimedia Tools and Applications, 2016. 75(23): p. 15601-15617.

[31] Lopes, N.V., do Couto, P.A.M., Bustince, H. and Melo-Pinto, P., 2010 Automatic histogram threshold using fuzzy measures. IEEE Transactions on Image Processing, 19(1), pp.199-204.

[32] Gao, H. and Chae, O., 2010. Individual tooth segmentation from CT images using level set method with shape and intensity prior. Pattern Recognition, 43(7), pp.2406-2417.

[33] Lung, J.W.J., et al., Fuzzy phoneme classification using multi-speaker vocal tract length normalization. IETE Technical Review, 2014. 31(2): p. $128-136$.

[34] Sharifara, A., M.S.M. Rahim, and M. Bashardoost. A novel approach to enhance robustness in digital image watermarking using multiple bitplanes of intermediate significant bits. in Informatics and Creative Multimedia (ICICM), 2013 International Conference on. 2013. IEEE.

[35] Jabal, M.F.A., et al. A comparative study on extraction and recognition method of CAD data from CAD drawings. in Information Management and Engineering, 2009. ICIME'09. International Conference on. 2009. IEEE.

[36] Harouni, M., et al., Online Persian/Arabic script classification without contextual information. The Imaging Science Journal, 2014. 62(8): p. 437-448. 